\title{
Investigation of the AFM Contact-Mode Force Calibration with Simulation
}

\author{
Judit Kámán, Attila Bonyár \\ Department of Electronics Technology, Budapest University of Technology and Economics, Budapest, Hungary \\ kaman@ett.bme.hu
}

\begin{abstract}
Force-curves measured by Atomic Force Microscopy (AFM) are frequently used to determine the local Young's Modulus of the sample. Originally the AFM (Atomic Force Microscopy) instruments measure the cantilever deflection as a voltage signal; however the natural unit of the deflection is nanometer. In general, the V/nm conversion factor is determined from the force-curve of a hard sample. Since this conversion is highly affects the value of the Young's modulus, the accuracy of this method was investigated experimentally and using a finite element simulation of the cantilever motion. It was found, that the position of the laser spot significantly modify the conversion factor and in case of the sample with steep surface, the error of this calibration method can be significant.
\end{abstract}

\section{INTRODUCTION}

Atomic Force Microscopy (AFM) is widely used to measure the surface properties of materials in the micron, sub-micron or even nanometer scale. Besides the most common topography mapping, a great advantage of AFM is that it can yield supplementary information concerning the physical properties of the surface, such as for example electrostatic, magnetic behavior, or mechanical (e.g. elastic) properties [1]. For the latter the so called force-curve evaluation - which is obtained by contactmode point-spectroscopy - is the most common approach [2]. In this technique the deflection of the cantilever during sample indentation in one spot is used to calculate the forces acting on the cantilever and thus in turn the elastic behavior of the surface. Most of the modern AFMs use the so called laserreflection based method to transform the deflection of the cantilever into a measurable signal (see Fig. 1 left). The laser light is reflected from the back side of the cantilever and is collected by a multi-segmented photodetector, which produces a voltage signal proportional with the deflection (Fig. 1).

The physical models, which are used to evaluate the obtained force-curves through fitting, such as the Herz-Sneddon model [2-4], JRK (Johnson-RobertsKendall model) [5], or the DMT (Derjaguin-MullerToporov model) [6], require the cantilever deflection in a metric dimension for the calculation of the elastic modulus (Young's modulus) of the sample $(\boldsymbol{E})$. For this reason a $\mathrm{V}$ to $\mathrm{nm}$ conversion is necessary on the obtained raw force-curve data to use it for subsequent processing. According to these mentioned contact mechanics models, which describe the tip-surface interaction, the force $(\boldsymbol{F})$ between the tip and the sample is proportional to the deformation depth $(\delta)$ of the sample to a positive power [7]. The deformation depth can be calculated as the difference between the sample motion $(z)$ and the cantilever deflection $(\boldsymbol{d})$ during the contact-mode point-spectroscopy (Eq. 1).

$F \sim \delta^{n}=(z-d)^{n}$, where $n>0$

It can be seen, that the value of the conversion factor $(\Delta z / \Delta \boldsymbol{V})$ severely influences the results of the force-curve processing. The Young's modulus can be obtained from the proportional factor by fitting the selected theoretical model on the measured $\boldsymbol{d}(\boldsymbol{z})$ curve. In practice the most common way to determine the V/nm conversion factor (also called the deflection inverse optical lever sensitivity, or DeflInvOLS [8]) is performing contact-mode point-spectroscopy measurements on an ideally hard substrate. The theoretical background is, that during pointspectroscopy, in the case of a hard material, the deflection of the cantilever in $\mathrm{nm}(\boldsymbol{d})$ ideally equals the movement of the tip in the $\mathrm{Z}$ dimension in $\mathrm{nm}(z)$, thus the gradient of the obtained point-spectroscopy curve is the inverse of the conversion factor (Fig. 1.). Since 
this method can be harmful for the tip the users sometimes do it at the end of the measurements.
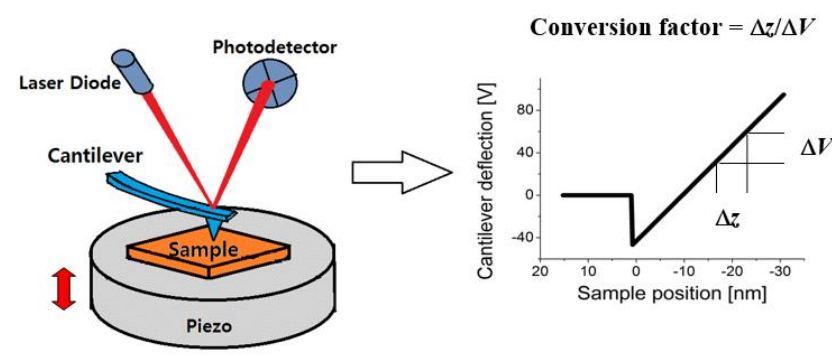

Fig. 1. Illustration of the laser-reflection based method to determine the cantilever's deflection (left) and the measured signal during point-spectroscopy (right)

The weakness of this method is that is presumes several factors to be ideal, e.g. that the tip movement $(z)$ and cantilever deflection $(d)$ is linear in the whole investigated range (which is usually not), or that only normal forces act on the cantilever (lateral forces, which could cause torsion in the cantilever are neglected), which presumes ideal contact between the tip and the surface. Another neglected factor is the effect of the position of the laser beam on the backside of the cantilever. Since the deformation (due to deflection) of the cantilever is not uniform along its length and since the focus diameter of the laser spot is comparable with the dimensions of the cantilever, its position can significantly reflect on the measured slope of the point-spectroscopy curve.

The exact position of the laser spot is often neglected or changed during or between measurements (due to for example recalibration of the detector signal). Since the obtained conversion factors directly influence the determination of the Young's modulus during force-curve processing, it would be important to know how much effect the position of the spot can have on the results. For this reason, in our work we aim to investigate the effect of the laser spot position on the measured conversion factor, both experimentally and through finite element modeling.

\section{METHODS}

\subsection{Simulation}

The bending of the cantilever was simulated using finite element method to investigate the shape of the cantilever under a specified loading force. The twodimensional plane stress model was used in a custom Matlab code based on [9]. The cantilever was considered as a homogeneous, isotropic material (the nominal geometrical values provided by the supplier, see Section 2.2.), and the four-node quadrilateral element was applied in the mesh using twodimensional Lagrange shape functions. The effect of the tip was considered in the distribution of the loading force and in constrains. The approaching part of the contact-mode point-spectroscopy was simulated applying $500 \mathrm{nN}$ maximum loading force on the cantilever.

\subsection{Experimental}

A Veeco diInnova type atomic force microscope (AFM) was used for the measurements. The force curves were obtained by performing contact-mode point-spectroscopy with an ART D160 diamond probe on a silicon surface. The nominal values of the tip were used for the subsequent the calculations, namely the spring constant $(k)$ was regarded as $5 \mathrm{~N} / \mathrm{m}$ and 10 degree was used as the half opening angle of the tip $(\alpha)$. The Poisson-ratio of the silicon was assumed to be $v=0.27$. The nominal values of the cantilever geometrical parameters are the following; length is $125 \mu \mathrm{m} \pm 5 \mu \mathrm{m}$, width is $25 \mu \mathrm{m} \pm 3 \mu \mathrm{m}$, thickness is 2 $\mu \mathrm{m} \pm 0.5 \mu \mathrm{m}$. For the simulation the mean values were used. $E=179 \mathrm{GPa}$ was used for the simulation as the elastic modulus of the cantilever.

Fig. 2 presents a schematic illustration of the investigated laser spot positions. The four positions were selected to be equidistant from the tip of the cantilever. The position of the spot was estimated based on the optical microscopy images made on the cantilever during the measurements. The centers of the four positions were $22 \mu \mathrm{m}, 49 \mu \mathrm{m}, 87 \mu \mathrm{m}$ and $109 \mu \mathrm{m}$ away from the base of the cantilever (the total length of the cantilever is $125 \mu \mathrm{m}$ ). These values were also used for the simulations.

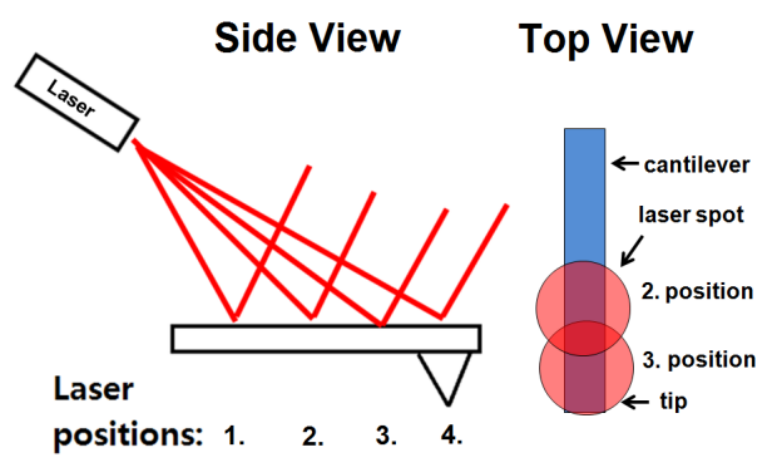

Fig. 2. Schematic illustration of the investigated laser spot positions on top of the cantilever. 


\section{RESULTS AND DISCUSSION}

Figures 3-5 present the measurement and simulation results, respectively. The slope of the point-spectroscopy curves were measured in function of the position of the laser spot atop the cantilever. This measured slope of the curves $(\Delta \boldsymbol{V} / \Delta \boldsymbol{z},[\mathrm{V} / \mathrm{nm}]$, see Fig. 3) is the inverse of the conversion factor $(\Delta z / \Delta V,[\mathrm{~nm} / \mathrm{V}])$, which is used to transform the pointspectroscopy curves into force-curves. As can be seen in Figs. 3 and 4, the measured slope is significantly influenced by the position of the laser spot.

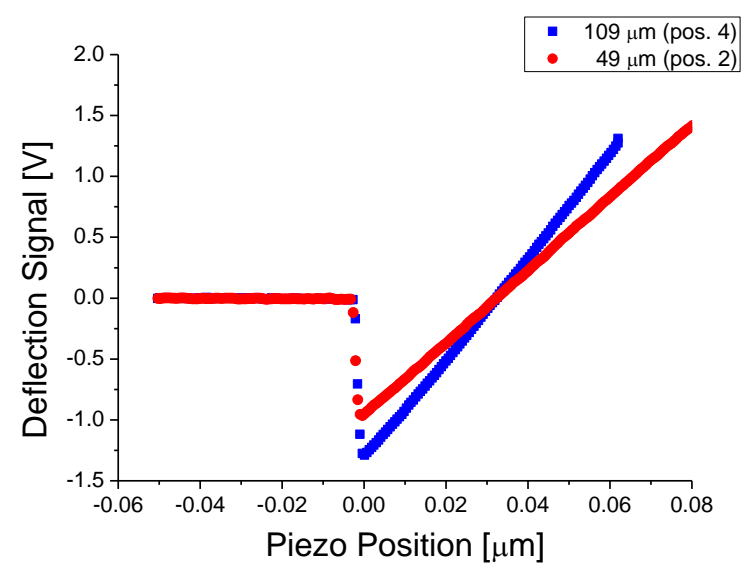

Fig. 3. The measured point-spectroscopy curves obtained at 49 and $109 \mu \mathrm{m}$ from the base of the cantilever, respectively.

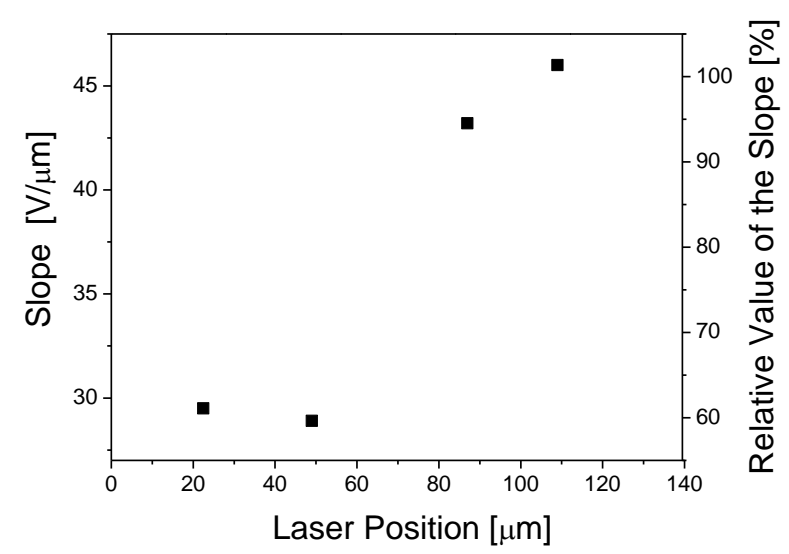

Fig. 4. The measured slope of the force curves in function of the position of the laser spot on top of the cantilever (see

Fig 2). Relative values are given considering the value measured at $109 \mu \mathrm{m}$ as $100 \%$.

By comparing the measured data with the simulation results presented in Fig. 5, we can see, that the characteristic of the curves match. However, the simulation results suggest a stronger dependence of the measured slope on the position of the spot. This significant difference could arise either from the imperfection of the model, or from the fact, that the model neglects the effect of the photodetector, since it only calculates the slope as a linear shift of the reflected laser beam from the cantilever. In the real measurements this beam is reflected into a foursegmented PSPD (position sensitive photo detector), where the linear shift of the spot is transformed into voltage, based on the position of the beam in the segments. Although the manufacturer generally states how the signal is calculated (as a difference between the top and lower segments), we do not have detailed information about the precise linearization and normalization algorithms they probably use to generate the $\pm 10 \mathrm{~V}$ ranged output signal. Besides, the model presumes that only normal forces act on the cantilever. During the real measurements it could have been possible that due to an improper contact between the tip and the surface lateral forces also occurred (these lateral forces were not monitored during the measurements), which could cause differences in the measured slopes.

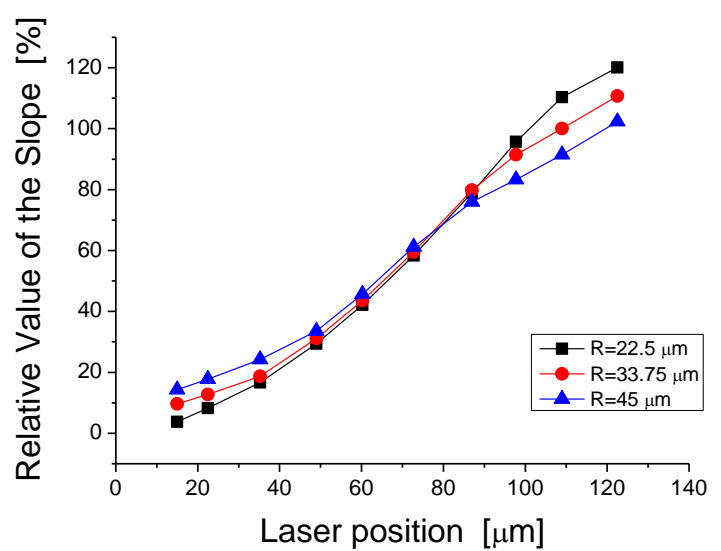

Fig. 5. Relative values of the slope obtained by simulations with different laser spot radius $(22.5,33.75$ and $45 \mu \mathrm{m})$, in function of the position of the laser spot on top of the cantilever (see Fig 2). Relative values are given considering the value obtained at $109 \mu \mathrm{m}$ with $\mathrm{R}=33.75 \mu \mathrm{m}$ laser spot radius as $100 \%$.

Regarding the significance of the results, the following can be stated. In AFM practice the laser is generally positioned at the end of the cantilever, close to the tip. Considering only this end of the obtained curves (120-90 $\mu \mathrm{m}$ range), the relative sensitivity of the slope (and thus the determination of the conversion factor) is around $0.5 \% / \mu \mathrm{m}$ for the measured data and around $1 \% / \mu \mathrm{m}$ for the simulations. This means that 
for example a $10 \mu \mathrm{m}$ repositioning of the spot on top of the cantilever can possibly cause a 5-10\% difference in the obtainable conversion factor. Since it directly affects the scaling of the force curves and the elastic modulus depends in non-linear way on it, in turn a $15-20 \%$ difference in the measured Young's modulus. In other words, repositioning the laser spot between calibration and measurements could cause significant error in the scaling of the obtained forcecurves, which could lead to variance in the calculated elastic modulus.

Besides the investigated phenomenon, there are several other aspects of the practice of force-curve measurements which could cause similar of even higher inaccuracy in the determination of the elastic properties of the samples. In order to maximize precision, the position of the spot should not be changed between cantilever sensitivity calibration and the measurements.

\section{Conclusions}

The calibration method which was used to scale AFM point-spectroscopy data was investigated experimentally and by finite element method based simulations. It was confirmed, that the conversion factor (cantilever sensitivity) is strongly depending on the position of the laser spot on top of the cantilever. The relative sensitivity of this position dependence is found to be between $0.5-1 \% / \mu \mathrm{m}$. It can highly affect the elastic modulus because the Young's modulus is derived from the force curve that is rescaled by the application of this conversation factor. Results indicate that the repositioning of the laser spot between cantilever sensitivity calibration and measurements are not advised.

\section{ACKNOWLEDGEMENTS}

The research leading to these results has received funding from the ProProgressio foundation. Attila
Bonyár is grateful for the support of János Bolyai Scholarship.

\section{REFERENCES}

[1] P. Eaton, P. West, Atomic Force Microscopy, Oxford University Press, 2010.

[2] Butt, H. J., Cappella, B., Kappl, M. "Force measurements with the atomic force microscope: Technique, interpretation and applications." Surface Science Report. 59 (1-6). pp. 1-152. 2005.

[3] Herzt, H. "Ueber die Berührung fester elastischer Körper." (The contactof solid elastic bodies.) Journal für die reine und angewandte Mathematik. pp. 156-172. 1882. (In German)

[4] Domke, J., Radmacher, M. "Measuring the elastic properties of thin polymer films with the AFM." Langmuir. 14. pp. 3320-3325. 1998.

[5] Johnson, K. L., Kendall, K., Roberts, A. D. "Surface energy and the contact of elastic solids." Proceedings of the Royal Society of London, Series A. 324. pp. 301313. 1971.

[6] Derjaguin, B. V., Muller, V. M., Toporov, Y. P. "Effect of the contact deformation on the adhesion of particles." Journal of Colloid and Interface Science. 53. pp. 314-326. 1975.

[7] D. C. Lin, E. K. Dimitriadis, F. Horkay " Robust strategies for automated AFM force curve analysis--I.", J. Biomech. Eng., vol. 129, no. 3, pp 430-440, 2007

[8] Y.-R. Chang, V. K. Raghunathan, S. P. Garland, J.T. Morgan, P. Russell, C. J. Murphy, "Automated AFM force curve analysis for determining elastic modulus of biomaterials and biological samples", jornal of the Mechanical Behviour of Biological Materials, 37(2014) pp. 209-218

[9] A. J. M. Ferreira, MATLAB Codes for Finite Element Analysis: Solids and Structures (Solid Mechanics and Its Applications), ISBN: 978-1-4020-9199-5 (Print) 978-1-4020-9200-8 (Online), Springer (2009) 